\title{
Latest Trends in Civil Engineering
}

\author{
GKV Ramachandran* \\ DNV GL Renewables Certification, Denmark
}

*Corresponding author: GKV Ramachandran, DNV GL Renewables Certification, Denmark.

Received Date: October 01, 2018

Published Date: October 10, 2018

\section{Opinion}

Latest developments in civil engineering will have a tremendous impact in the coming years as this field of core engineering is spanning from the day to day life of the society such as the field of infrastructure, which include buildings, roads, bridges for instance to the more sophisticated fields such as aerospace, energy, transportation etc., In the last few decades the civil engineering land scape has been undergone many changes due to the advent of computers and computing power, wherein the designs were moved from physical drawing boards to the computer screens. The designs were impacted beyond imagination as the three-dimensional (3D) viewing software packages became accessible to the design firms so that the users could view, feel, and even could provide suggestions before the designs were implemented and built. This impact was not only on the designs, but also tremendously on the analysis part, wherein the designs went through several iterations and finally ended up in optimum designs resulting huge cost saving. Having said a brief history on the last few decades, what could be driving the forthcoming years' developments? Without doubt, we can say the magic word DATA, which is going to influence the recent and future developments in civil engineering, similarly to many other fields. Data together with core civil engineers, data scientists, computer engineers, and statisticians could change the outlook of today's civil engineering. No wonder the Industry 4.0 revolution will certainly impact not only on all the fields of engineering, but also on all other fields such as Medicine (e.g. diagnostics, medicine identification, etc.), Law (e.g. IBM Watson can read millions of documents in seconds of time, which would help legal practitioners to find the correct law points), etc.,

Even though what is said above would look futuristic, the hard reality is that it is happening already. For that, if we analyze topic by topic, it will become more clear. As a first example, if we take Bridges, already Drones are being used for inspections to detect faults, cracks, etc., If the environmental, boundary conditions, and bridge inspection data (need not be drone monitored one, it could be manual inspection, for example) for the similar bridge designs are available, then with the acquired inspection data, Analytics tools can analyze the data and with Machine learning algorithms, it can provide lot of prescriptive information - for example, by predicting the local environmental and boundary conditions, it can provide what would be the health condition of the bridge. Based on this information, future inspections, or repairs, or even retrofitting of the bridges would be possible. This would save lot of time, energy, and money. On top of all this, safety of the people can be ensured by predicting failure conditions. Terminology for dealing with huge data is known as Big Data and as the storage capacity needed is proportionally large, Engineers and Scientists dealing with big data is making use of Cloud services, such as Microsoft Azure for instance. As we discussed about using drones for inspection, there will also be sensor-embedded designs, wherein the health condition of the bridges will be acquired instantaneously and stored in cloud and used for further processing and decision making. This technology is termed as Internet of Things (IoT). Assemblage of the above technologies is known as Digitalization, which is picking up rather fast, and would become more matured in a few years' time and will be more popular with the ensuing $5 \mathrm{G}$ connectivity.

While the above case study showed the impact of the Digitalization on one specific area, generically any product can be termed as an Asset, as the product is having certain value and it has a certain life span as well. Hence, managing an Asset in a smart way can probably lead to its life time extension, thereby higher returns than expected. This is one of the main motivations of the investors to support these developments. Along with the advantages, it is also important to mention a few pit falls as well. Because, for all these smart tools, involvement of a domain expert is inevitable in order to choose an appropriate algorithm or a statistical method by a data scientist. Otherwise, completely opposite results may arise, which could cause more damages than any savings. Further developments include the application of Artificial Intelligence (AI), Additive Manufacturing, 3D printing, etc., AI will have a huge impact on transportation engineering. One popular example is the driverless vehicles. For the road transportation, this new technology is termed as Intelligent Transportation System (ITS), which is a collaborative technology between transportation, 
computer science, electronics and communication disciplines along with many others. Implementation of this would need exclusive roads, which would use many sensors and the technology to mobile charge the vehicles. This would optimize todays' traffic chaos in certain parts of the world, ensure much more efficiency, carbon emission reduction as these would be powered by electric vehicles using cleaner technologies such as Wind, Solar etc.,

To summarize, the rapid growing digitalization developments and cleaner technologies would revolutionize the conventional engineering disciplines including civil engineering in order to help the world to be more green and sustainable.

\section{Acknowledgment}

None.

\section{Conflict of Interest}

No conflict of interest. 